\title{
Acute Management of Anticoagulation-Associated Intracerebral Hemorrhage
}

\section{(c) (1) $\ominus$}

Authors

Joji B. Kuramatsu, Stefan T. Gerner, Hagen B. Huttner, Stefan Schwab

Affiliation
University Hospital Erlangen - Department of Neurology

Key words

intracerebral hemorrhage, anticoagulation, hematoma enlargement, blood pressure reductions, reversal

\author{
Bibliography \\ DOI https://doi.org/10.1055/s-0043-108836 \\ Neurology International Open 2017; 1: E275-E286 \\ (c) Georg Thieme Verlag KG Stuttgart - New York \\ ISSN 2511-1795
}

Correspondence

Joji B. Kuramatsu

University Hospital Erlangen

Department of Neurology

Schwabachanlage 6

91054 Erlangen

joji.kuramatsu@uk-erlangen.de

\begin{abstract}
The incidence of anticoagulation-associated intracerebral hemorrhage $(\mathrm{OAC}-\mathrm{ICH})$ is increasing along with demographic changes and patients requiring oral anticoagulation for atrial fibrillation. OAC-ICH is one of the most detrimental sub-types of hemorrhagic stroke, characterized by larger hemorrhage volumes, more frequent intraventricular hemorrhage, increased and prolonged hematoma enlargement. The impact of these outcome predictors' results in greater mortality rates and larger numbers of patients left in functionally dependent states after ICH. Yet, evidence on treatment options for these severely affected patients remains limited, resulting in weak recommendations by international and European guidelines. Minimization of hematoma enlargement constitutes the main
\end{abstract}

focus of acute care, possibly achieved by blood pressure and reversal management. In all OAC-ICH patients irrespective of anticoagulation agent, immediate and aggressive blood pressure reductions as fast as possible targeting systolic blood pressure levels of $140 \mathrm{mmHg}$ (at least within 4-6 h) seems reasonable. Specifically, in vitamin-K-associated ICH, blood pressure reductions have been shown to be associated with reduced rates of hematoma enlargement. Reversal management in OAC-ICH should be carried out as quickly as possible, though accounting for the different anticoagulation agents used. In vitamin-K-associated ICH, recent large-sized multicenter investigations contributed to refining reversal strategies. One German-wide cohort study including roughly 1200 patients identified for the first time beneficial target values to guide reversal treatment. Anticoagulation reversal should achieve INR values of less than 1.3 or 1.2 at least within $4 \mathrm{~h}$ after admission. Agents to be used comprise prothrombin complex concentrates (PCC), which may achieve faster and more complete reversal. This combined approach (blood pressure management and INR-reversal) was associated with significant reductions of hematoma enlargement and lower in-hospital mortality rates. For dabigatran-related bleeding complications, most recently an antidote (Idarucizumab) has been approved, which has been prospectively investigated and showed rapid and almost complete reversal as well as sufficient hemostasis. Currently, bleeding under the influence of factor-Xa inhibitors poses great difficulties because no antidote is available and efficacy of commonly used antagonizing agents, i. e., PCC or fresh-frozen plasma (FFP), has not been proven for clinical use. The main aim of the present review focuses on acute-care management of OAC-ICH and tries to depict treatment options based on the most current data. Moreover, current neurocritical-care therapies for ICH will be reviewed to identify relevant implications specific to OAC-ICH treatment.

\section{Epidemiology \& Etiology}

One of the most serious forms of stroke is intracerebral hemorrhage (ICH) as fewer than $30 \%$ of patients recover to functional independence and the one-year mortality rate of over $50 \%$ is very high [1-3]. In hemorrhagic stroke, oral anticoagulation-associated intracere- bral hemorrhage $(\mathrm{OAC}-\mathrm{ICH})$ is of particular concern because it is associated with larger hemorrhage volumes, increased ventricular extensions, and more frequent and protracted hematoma enlargement [4-7]. All of these parameters are validated for a negative prognosis, which is even poorer considering the patient character- 
istics that necessitated oral anticoagulation in the first place $[1,8-$ 10]. These patient characteristics usually include advanced age and a pronounced cerebrovascular risk profile and are also associated with a high risk of hemorrhage and thromboembolism [1, 8-11]. Currently the European and international guidelines offer very few evidence-based treatment recommendations for these critically ill patients $[1,8,12,13]$.

In Germany, the incidence of hemorrhagic stroke is currently about 50 per 100000 patient-years and falling (between 1990 and 2010), and the mortality rate has dropped by nearly half in this time period [14]. Of the total number of ICHs, approximately $15-25 \%$ is related to oral anticoagulation $[10,15,16]$. The incidence of OAC$\mathrm{ICH}$ is therefore approximately 10 per 100000 patient-years in Europe, and anticoagulants increase the risk of hemorrhage almost tenfold $[7,17]$. Epidemiological studies indicate that the incidence of ICHs in the western hemisphere has been relatively stable or has fallen over the last several decades. In contrast, OAC-ICHs and the atypical ICHs discussed below are of special significance because the incidence of these hemorrhage entities is expected to increase $[2,14,18]$. Due to their localization, intracerebral hemorrhages are generally categorized as typical or atypical and presumed to have differing pathoetiogeneses. The typical (or "loco typico") ICH is limited to the basal ganglia region, in some cases with involvement of the thalamus. The primary genesis of this form of hemorrhage is hypertension, because the resulting vascular damage causes the striatal vessels to rupture. An atypical hemorrhage is lobar and characterized by the involvement of cortical and subcortical structures [2]. This form of ICH has particular characteristics and is often associated with cerebral amyloid angiopathy (CAA), which in itself is characterized by multiple microbleeds and more frequently recurring macrobleeds $[2,19,20]$. This vulnerability to hemorrhage may represent a relevant association or even a theoretical predisposition for the occurrence of an oral anticoagulant-associated $\mathrm{ICH}$. The increase in atypical ICHs could therefore be associated with the increasing incidence of atrial fibrillation and the resulting more frequent use of anticoagulants alongside the demographic change $[2,18,20,21]$.

For patients aged 65 and older, the incidence rate of an ICH without oral anticoagulation is the lowest, at approximately $0.3 \%$ per year [14]. In the global context, high-income countries should be differentiated from low- and middle-income countries, because the ICH incidence rate in the latter is more than twice as high and significantly increasing [14]. For patients under oral anticoagulant therapy, the most relevant difference is the form of oral anticoagulation. For patients taking vitamin $\mathrm{K}$ antagonists (VKA), the annual incidence rate is approximately $1 \%$ compared to patients taking novel oral anticoagulants (NOAC) whose incidence was half that [22]. It should be noted, that the data used was evaluated within the framework of the major NOAC clinical trials $(n=10000)$ and reflects a selected patient cohort. "True" clinical data suggests that the incidence of OAC-ICH in patients who have never suffered an intracranial bleed is probably somewhat higher - approximately 1-2\% [23]. The presumed largest relative increase in the incidence rate is evident in patients who have already suffered an $\mathrm{ICH}$ and are treated with anticoagulants due to the high risk of thromboembolism. For this patient cohort, the weight of evidence is not optimal, although possible incidence rates of up to $10 \%$ per year are assumed $[10,15,24-28]$.

\section{Risk Factors for an OAC-ICH}

Due to similar risk factors, the risk for hemorrhagic complications rises basically in parallel with the risk of thromboembolism. Validated ischemic risk scores (CHADS2, CHA2DS2-VASc) provide a way to assess the risk of hemorrhage. Hemorrhage-specific scores (HAS-Bled, HEMORR2HAGES) that account for conventional risk factors, liver and kidney function problems, and taking anticoagulant medications allow for a more precise estimate of hemorrhage risk $[9,11,29,30]$. For patients taking VKA anticoagulants, a correlation was demonstrated between the degree of anticoagulation, as measured by the INR, and the probability of occurrence of a hemorrhagic stroke. In the supratherapeutic INR range, the risk of hemorrhage rises dramatically, i. e., more than fivefold at an INR between 4 and 4.5 and nearly twentyfold at an INR greater than 4.5. For patients taking NOACs, a similar correlation, e. g., between the plasma concentration of the given preparation and the probability of occurrence of an $\mathrm{ICH}$, could not yet be demonstrated [31]. However, this could be due to the lack of internationally uniform and standardized level-measurement procedures and the relatively short time these medications have been in daily clinical use. The patient's age is a major factor associated with the occurrence of extra- and intracranial bleeds in patients under oral anticoagulant therapy. Starting at the age of 35, the patient's risk of the incidence of ICH doubles every ten years [32]. Explicitly for patients under oral anticoagulant therapy, a post-hoc analysis of the ROCKET-AF data showed an independently significant association between patient age (increase of 10 years) and the occurrence of an intracranial hemorrhage (hazard ratio: 1.35 (1.13-1.63), $p=0.001$ ) [33]. Similarly, data from the RE-LY trial also showed an independent association between patient age (age $>75$ years) and serious bleeding complications [34]. In a prospective study of just under 500 patients recently started on oral anticoagulation with vitamin $\mathrm{K}$ antagonists, there was also an age-dependent difference in the bleeding incidence over the course of 1 year (age $\geq 80$ years: 13.1 versus age $<80$ years: 4.7 ; per 100 person-years $p=0.009$ ) [23].

Not only oral anticoagulation alone, but additive medications influencing coagulation may even further increase the risk of recurrent hemorrhage. Dual therapy with antiplatelet drugs and oral anticoagulation is of particular concern in this regard. Available data from Germany indicates that, at least in patients who have suffered an $\mathrm{OAC}-\mathrm{ICH}$, approximately $11 \%$ were taking platelet function inhibitors at the same time [10]. Randomized data showed that the bleeding incidence from added antiplatelet drugs at least doubled the intracranial bleed complication rate with simultaneous oral anticoagulation [35]. There have been no studies to date examining such dual therapy in patients who have suffered a cerebral hemorrhage. Yet a leading medical journal reported on a study of over $1000 \mathrm{ICH}$ patients, and albeit the main purpose was to evaluate the influence of long-term blood-pressure control after ICH, there do appear to be independent effects of platelet function inhibition on ICH recurrence [27]. In this study, Biffi et al. clearly demonstrated this independent association after lobar ICH in that the risk of recurrent hemorrhage under platelet inhibitor therapy was nearly three times higher (hazard ratio $2.89(1.32-6.30)$; $p=0.008)$. For patients with non-lobar $\mathrm{ICH}$, this unfavorable influence was less pronounced (hazard ratio $1.56(0.98-2.48) ; p=0.06)$. For both hemorrhage entities, the most important result of this study was 
the consistent and independent influence of poorly adjusted antihypertension therapy with rebleeding rates (blood pressure: systolic $>140 \mathrm{mmHg}$, diastolic $>90 \mathrm{mmHg}$ ) in the long-term course post-ICH. Not only could the independent influence of poorly controlled hypertension on the recurrent hemorrhage rate be established, but there was also a correlation between blood pressure adjustment and bleeding risk, i. e., the higher the systolic blood pressure (in $10 \mathrm{mmHg}$ intervals), the higher the probability of occurrence of another $\mathrm{ICH}$.

Another factor that is related to the increased occurrence of $\mathrm{ICH}$ is cerebral amyloid angiopathy (CAA). This disease is characterized by excess deposits of beta-amyloid in the small and medium-sized blood vessels in the cortical and subcortical regions as well as the leptomeningeal vessels. Histologic examination is required for definitive diagnosis, however further imaging tests (MR: T2, SWI, GRE sequences) can be used to diagnose a probable CAA [36]. In CAA, the white matter, the deep gray matter and the brain stem are generally spared from microbleeds. Microbleeds can also be present in deep $\mathrm{ICH}$, but then are restricted to the basal ganglia associated with a hypertensive vasculopathy. The morphological imaging correlates of CAA are multiple microbleeds in the lobar localization particularly in the temporal and occipital lobes versus the frontal and parietal lobes [19, 36]. Like Alzheimer's disease, the incidence of CAA appears to be correlated with age and there are clear associations between CAA and the increased occurrence of $\mathrm{ICH}$ and recurrent hemorrhage [37]. After age, anticoagulant medications appear to have a stronger influence, because an accumulation of microbleeds is found in ICH-naïve patients not only under anticoagulation, but also under antiplatelet drug therapy [38, 39]. Likewise, a comparison of the microbleed burden in patients who have suffered an ICH with and without anticoagulation showed a marked accumulation of microbleeds under antiplatelet and oral anticoagulation therapy $[38,39]$. Furthermore, CAA exhibits a genetic association with ApoE (epsilon 2 \& 4) alleles and is associated with a higher risk of re-bleeding in carriers of these alleles [40]. In an overall view of the $\mathrm{ICH}$ risk factors described, patients with lobar hemorrhage have a higher risk of recurrence (3.4\% versus $7.8 \%$ per year) than those with basal ganglia bleeds because they are usually older, have more frequent microbleeds and CAA, as well as increased comorbidities with a simultaneous indication for anticoagulant medications [27, 38, 39].

\section{Assessment of Prognosis and Specific Characteristics of OAC-ICH}

Compared to OAC-ICH, the validated prognostic parameters of primary ICH are identical, although there are specific differences in possible therapeutic approaches to acute treatment, which will be described in the following. Generally, all validated predictors of $\mathrm{OAC}-\mathrm{ICH}$ are more pronounced, leading to a poorer functional condition and a higher mortality rate after $\mathrm{OAC}-\mathrm{ICH}$. The validated prognostic parameters are: age, neurological status (GCS, NIHSS), hematoma volume, ICH localization, intraventricular hemorrhage, and hematoma progression.

As already discussed, patient age is a critical prognostic parameter in stroke patients and also significantly influences the func- tional condition of those who survive ICH. Accordingly, age is a component in all commonly used risk scores (e. g., ICH score, FUNC score) to prognosticate mortality and functional outcome [41-43]. In a prospective study, the influence and significance of the Glasgow Coma Scale (GCS) was investigated as a substitute for neurologic status in over $1000 \mathrm{ICH}$ patients to predict 30-day mortality [44]. Compared to the ICH score, the categorized GCS was similarly predictive (ICH score: area under the curve $=0.861$ versus GCS: area under the curve $=0.872$ ) [44]. The National Institutes of Health Stroke Scale (NIHSS) is the most important quantitative measurement of neurologic status after stroke and is explicitly stressed in the current American guidelines for ICH treatment [1]. In large stroke registry studies $(n>10000)$ including ICH patients, the strength and relevance of the NIHSS to predict prognosis was demonstrated, and the NIHSS also improved the predictive value of previous scores for ICH patients [45].

The most important prognostic parameter is the initial hematoma volume, and a volume greater or less than $30 \mathrm{ml}$ is a very frequently used threshold to assess prognosis and define inclusion criteria in randomized studies $[1,46,47]$. Hematoma localization also plays an important role. In supratentorial bleeds, the lobar ICH appears to be associated with a generally better outcome than basal ganglia bleeds, whereas infratentorial ICHs are associated with a poorer prognosis. Basal ganglia bleeds are accompanied significantly more often by intraventricular hemorrhage, which may lead to occlusion of the 3rd and 4th ventricle associated with an acute hydrocephalus, a consecutive rise in brain pressure and - in the worst case - herniation. Various studies have shown that not only does the presence of intraventricular hemorrhage worsen prognosis but so does the ventricular blood volume [4].

The hematoma progression is the most clinically relevant prognostic parameter because it seems to be generally treatable. It is important to differentiate between treatable and nonmodifiable parameters; patient age, initial hematoma volume, and neurologic status are not modifiable, whereas hematoma growth and intraventricular extensions are events that arise especially in the acute phase $(0-72 \mathrm{~h}$ ) and are amenable to treatment. These treatable parameters - hematoma growth and ventricular intrusion - play a special role particularly in the acute treatment of OAC-ICHs, because they occur much more frequently than in primary ICHs and represent an extremely relevant therapeutic target. The literature on OAC-ICHs describes not only a cumulative but also a prolonged occurrence of hematoma progression; furthermore, hematoma growth seems to be accompanied by repeat ventricular intrusion [4-6]. Additionally, intraventricular hemorrhage in OAC-ICH appears more frequently and is especially more pronounced. In summary, this treatment goal - avoiding hematoma growth and recurrent intraventricular hemorrhage- in OAC-ICHs represents a potentially very relevant treatment target in acute -ICH care that may be able to influence functional endpoints through targeted interventions.

Based on a post-hoc analysis of the randomized INTERACT 1 \& 2 studies, described in greater detail in the following, a prognosis model (BRAIN score) was developed to assess the risk of hematoma growth [48]. The model has a maximum point value of 24 , at which the probability of occurrence of hematoma growth was determined to be $86 \%$ with overall good discrimination (c-statistic, 
0.73). The following influencing parameters - ICH volume, previous $\mathrm{ICH}$, intraventricular hemorrhage, anticoagulation, and time window (onset of symptoms to diagnosis) - were identified and built into the model. Along with hematoma volume, the highest weighted parameters were existing anticoagulation (6 points) and time window ( $<1 \mathrm{~h}=5$ points) [48]. This illustrates the relevance of the time window not only in ischemic but also hemorrhagic stroke patients. On this basis, patients with a high risk for a prognosis-relevant complication may be able to be identified and treated early on. The time window - not only onset of symptoms to diagnosis but also diagnosis until start of antagonization and acute therapy - plays a major role especially in OAC-ICHs, because the treating physician can positively influence the parameters that are independently associated with hemorrhage progression [10].

\section{Acute Treatment Within the First $24 \mathrm{~h}$}

The main goal of acute treatment of OAC-ICH is to minimize the risk of hematoma expansion to prevent further secondary worsening due to ICH volume effects and intracranial pressure elevations ventricular intrusion with acute hydrocephalus, and finally herniation. As already discussed, complications from hematoma growth in OAC-ICHs are significantly more frequent than in spontaneous ICHs and can occur protractedly for up to $72 \mathrm{~h}$ after the initial event $[6,49]$. Current studies suggest that hematoma growth occurs in well over one-third of patients with OAC-ICH, whereby differences in the risk of hematoma growth between VKA- and NOAC-associated hemorrhaging remains unclear $[10,50]$. To avoid hematoma expansion, current guidelines recommend working toward rapid normalization of coagulation and effective lowering of blood pressure in the acute phase [1]. However, the guidelines do not yet include the target values cited in the current data for rapid normalization of coagulation of VKA-ICH (the specific time window and INR value).

\section{Lowering blood pressure}

There are currently multiple randomized studies that have investigated the significance of aggressively lowering blood pressure on outcome and hematoma progression in spontaneous ICHs. In the randomized INTERACT 1 pilot study [51] published in 2008, the effect of an intensive reduction in blood pressure (systolic target value $140 \mathrm{mmHg}$ ) versus the then recommendation in the guidelines (target value $180 \mathrm{mmHg}$ ) on hematoma expansion was investigated in 404 patients. A more aggressive reduction in blood pressure was associated with a lower proportional hematoma growth after $24 \mathrm{~h}$ (intervention group: $13.7 \%$ versus control group: $36.3 \%$; $p=0.04$ ) and the risk of hematoma growth (defined as $>33 \%$ ) was $36 \%$ lower compared to the control group $(p=0.05)$. No differences were noted with respect to undesirable side effects or complications [51]. The multicenter ATACH 1 trial investigated the influence of blood pressure reduction using nicardipine (to one of three target ranges: systolic blood pressure $110-139 \mathrm{mmHg} / 140-$ $169 \mathrm{mmHg} / 170-199 \mathrm{mmHg}$ ) on the rate of hematoma expansion and the extent of perihemorrhagic edema, and functional outcome after three months [52]. Although no clinical benefit was demonstrated, the trial was not designed to detect differences in efficacy but to prove the safety of the above-cited therapy concepts. The
ICH-ADAPT study [53] was able to allay initial safety concerns that aggressive intravenous blood pressure reduction could reduce cerebral blood flow in the perihemorrhagic penumbra and promote secondary neurological damage. The INTERACT 2 trial published in 2013 in the New England Journal of Medicine compared rapid blood-pressure lowering (target systolic value $<140 \mathrm{mmHg}$ ) with a moderate reduction (target value $<180 \mathrm{mmHg}$ ) in $2839 \mathrm{ICH}$ patients within six hours after onset of hemorrhage. The treating physician was responsible for selecting the antihypertensive medication administered. In nearly $90 \%$ of patients in the intervention group (compared to $43 \%$ in the control group), the administration of i. v. antihypertensives was necessary to achieve the target blood pressure values. The medication most frequently given was urapidil (32.5\%), followed by calcium-channel blockers (16.2\%). The primary endpoint (poor outcome defined as modified Rankin score of 3-6 after 90 days) was just missed (OR 0.87 for rapid blood-pressure lowering (95\% Cl: 0.75-1.01); $p=0.06$ ), although an ordinal shift analysis of outcome and quality of life suggests that rapid blood-pressure lowering is of benefit after three months [54]. The subanalysis of 964 of the study participants who underwent brain imaging after $24 \mathrm{~h}$ showed an effective minimization of hematoma growth, especially in those patients treated earlier and more aggressively. However, it should be noted that only approximately one third of the patients in the intensive-treatment group actually achieved the target blood pressure of $<140 \mathrm{mmHg}$ [54]. Also recently published in the New England Journal of Medicine, the ATACH 2 trial attempted to achieve the clinical endpoint through earlier, aggressive blood pressure reduction because of the increased risk of hematoma expansion in the early phase of ICH [55]. The target values $(110-139 \mathrm{mmHg}$ vs. $140-179 \mathrm{mmHg}$ ) were to be achieved within $4.5 \mathrm{~h}$ after onset of symptoms through intravenous administration of nicardipine. After the interim analysis produced negative results, the trial was discontinued prematurely at 1000 patients before reaching the target enrollment of 1280 [55]. Intensive blood-pressure lowering proved no more effective than the standard treatment (modified Rankin score 4-6 after three months: intensive blood-pressure lowering 38.7 vs. 37.7\%; RR 1.04 (0.85$1.27)$ ) and was associated with a greater number of serious adverse events - especially an increase in renal complications - during the three-month observation period (serious complications: intensive-treatment group 25.6 vs. $20.0 \%$ in the standard-treatment group; relative risk $1.30(95 \% \mathrm{Cl}, 1.00-1.69) ; \mathrm{p}=0.05)$. Nonetheless, a statistical advantage in support of a reduced rate of hematoma expansion was also observed in this trial for the intervention group (standard treatment 24.4 vs. $18.9 \%$ in intensive-treatment group; RR 0.78 (0.58-1.03); $\mathrm{p}=0.08)$. Compared to INTERACT 2, all patients in the ATACH 2 trial were treated earlier and more aggressively, so in fact the blood pressure values in the control group were at the level of the patients who received intensive blood-pressure lowering treatment in the INTERACT 2 trial. At systolic values less than $130 \mathrm{mmHg}$ - like those achieved in the treatment group in ATACH 2 (mean value $128 \mathrm{mmHg}$ in the treatment arm) - it is possible that the side effects outweigh the positive effects on hematoma expansion. Caution is advised with respect to comparability and applying study results to German clinical practice and OAC-ICH. Unlike in the INTERACT 2 trial, the ATACH 2 trial excluded patients with $\mathrm{OAC}-\mathrm{ICH}(\mathrm{INR} \geq 1.5)$ and the antihypertensive was 
restricted to nicardipine - a calcium-channel blocker that is not approved for use in Germany, and due to its strong vasodilatory effect, is associated with cardiovascular and CNS side effects (including increased intracranial pressure) $[55,56]$.

Currently, the German-wide multicenter observational study RETRACE offers the most valid data basis for the value of blood pressure management in $\mathrm{OAC}-\mathrm{ICH}$; the study investigated both acute management as well as resumption of OAC after hemorrhage under treatment with marcumar [10]. The study conducted at 19 neurovascular centers included 1176 patients with marcumar-ICH defined as INR > 1.5 on admission; 853 patients were available for analysis of hematoma expansion via brain imaging. Blood pressure values were recorded at 4 -h intervals over the first $24 \mathrm{~h}$ after admission and tiered analysis performed in $20 \mathrm{mmHg}$ intervals. In the adjusted multivariate analysis, a systolic blood pressure of $<160 \mathrm{mmHg}$ within $4 \mathrm{~h}$ after admission was associated with a significantly reduced rate of hematoma expansion $(33.1 \%$ vs $\geq 160 \mathrm{mmHg} 52.4 \%$; $\mathrm{p}<0.001)$. To date, there have been no NOAC-ICH study results published that analyzed the value of blood pressure reduction. Finally, as-yet unpublished data for just under $200 \mathrm{NOAC}-\mathrm{ICH}$ patients in connection with a multicenter follow-up project of the German RETRACE trial (over 2500 OAC-ICH patients) indicate a similar relationship between blood pressure reduction and reduced hematoma expansion (Gerner et al., in revision). As a whole, the current data suggests that aggressive and early hemorrhage management (systolic blood pressure target of $140 \mathrm{mmHG}$ as quickly as possible, but at least within 4-6h), even in patients with altered coagulation, is a safe and effective way to prevent hematoma expansion, whereby a forced blood pressure reduction lower than the above-mentioned target values should be avoided.

- The goal of minimizing the risk of hematoma expansion is aggressive intravenous reduction of systolic blood pressure targeting $140 \mathrm{mmHg}$.

- As quickly as possible!

- However, hypotonic values (mean arterial pressure less than $80 \mathrm{mmHG}$ ) should be avoided in order to maintain cerebral perfusion should there be any intracranial hypertension.

\section{Antagonization VKA-ICH}

Normalizing coagulation is the crucial starting point in acute treatment of OAC-ICH. Prior to anticoagulant reversal, however, the given substance should be checked for effective administration ( Table 1). In VKA-ICH, anticoagulant reversal can be achieved by administering coagulation factors - such as fresh frozen plasma (FFP) or coagulation concentrates (PCC, prothrombin complex concentrates) - as well as providing vitamin $\mathrm{K}$. In a randomized, plasma-controlled, phase IIIb trial, the coagulation of 202 patients with vitamin-K antagonist-associated hemorrhage, of whom only 24 had an intracranial bleed, was corrected more quickly with a combination of vitamin $\mathrm{K}$ and PCC than with FFP (INR $\leq 1.330$ min after end of infusion: $\mathrm{PCC}+$ vitamin $\mathrm{K} 62.2 \%$ vs. $9.6 \% \mathrm{FFP}+$ vitamin $\mathrm{K}$ ) [57]. In another phase III study, vitamin-K antagonist patients $(n=181)$ with an INR $\geq 2.0$ and acute indication for surgical intervention were randomized and treated either with PCC (INR 2-4: 25 IU/kg KG; INR 4-6: $35 \mathrm{IU} / \mathrm{kg}$; INR > 6: $50 \mathrm{IU} / \mathrm{kg}$ ) or FFP (INR 2-4: $10 \mathrm{ml} / \mathrm{kg}$; INR 4-6: $12 \mathrm{ml} / \mathrm{kg}$; INR >6: $15 \mathrm{~m} / \mathrm{kg}$ ), each in combination with vitamin $\mathrm{K}$ [58]. In the PCC group, the surgical intervention could begin sooner after infusion started (PCC: $3.6(1.9-10.8)$ $h$ vs. $8.5(2.8-18.7)$ h in the FFP group; $p=0.0098)$ and more frequently with coagulation normalized (PCC $90 \%$ vs. FFP $75 \%$ ).

The only randomized, controlled study (INCH) published to date investigated the hemostatic potency of the different preparations in patients with VKA-ICH [59]. Fifty-four patients with VKA-ICH (INR $\geq 2$ on admission; inclusion within $12 \mathrm{~h}$ after onset of symptoms) were randomized and treated either with PCC (30 IU/ kg; $\mathrm{n}=27)$ or FFP $(20 \mathrm{ml} / \mathrm{kg} ; \mathrm{n}=23)$ combined with vitamin $\mathrm{K}(10 \mathrm{mg}$ i.v.). In addition to the normalization of coagulation (INR $\leq 1.2$ within $3 \mathrm{~h}$ after start of treatment), the study also included clinical endpoints such as rate of hematoma expansion and functional outcome at 90 days. The trial was terminated after inclusion of 50 of the 74 initially planned patients due to safety concerns in the FFP treatment group. PCC effectively normalized coagulation far more frequently (INR $\leq 1.2$ within $3 \mathrm{~h}$ : PCC $67 \%$ vs. FFP 9\%; OR 30.6 (4.7197.9); $p=0.0003)$. After $24 \mathrm{~h}$, the patients in the FFP treatment group evidenced greater hematoma expansion (ICH volume differ-

- Table 1 Reversal management in oral anticoagulation associated ICH.

\begin{tabular}{|c|c|c|c|}
\hline & \multirow[t]{2}{*}{ VKA } & \multicolumn{2}{|l|}{ NOAC } \\
\hline & & Dabigatran & Factor Xa inhibitors \\
\hline Indication & $I N R \geq 1.3$ & $\begin{array}{l}\text { Ingestion }<3-5 \text { terminal }{ }^{*} \text { half-life times } \\
\text { or Hemoclot }>30 \mathrm{ng} / \mathrm{ml} \text { ( }{ }^{*} \text { no renal } \\
\text { insufficiency) }\end{array}$ & $\begin{array}{l}\text { Ingestion }<3-5 \text { terminal } \\
\text { half-life times or calibrated } \\
\text { anti-XA level }>30 \mathrm{ng} / \mathrm{ml}\end{array}$ \\
\hline Acute treatment & $\begin{array}{l}\text { PCC [57] } \\
\text { INR 2-3.9-25 IU } / \mathrm{kg} \\
\text { INR } 4.0-6.0-35 \mathrm{IU} / \mathrm{kg} \\
\text { INR }>6.0-50 \mathrm{IU} / \mathrm{kg} \\
\text { If needed, re-administer after INR check }<4 \mathrm{~h} \\
\text { simultaneous i. v. vitamin } \mathrm{K}(10 \mathrm{mg})\end{array}$ & $\begin{array}{l}\text { Idarucizumab } \\
\text { If available } \\
\text { Otherwise PCC } \\
50 \mathrm{IU} / \mathrm{kg} \\
\text { Active charcoal (within } 4 \mathrm{~h} \text { ) if needed }\end{array}$ & $\begin{array}{l}\text { PCC/FEIBA } \\
50 \mathrm{IU} / \mathrm{kg} \\
\text { Active charcoal (within } 4 \mathrm{~h} \text { ) if } \\
\text { needed }\end{array}$ \\
\hline
\end{tabular}


ence $16.4 \mathrm{~mL}$, (95\% Cl: 2.9-29.9; $\mathrm{p}=0.018)$ and were at four times greater risk of hematoma expansion defined as a $>33 \%$ increase in volume over the initial volume, or death. The rate of thromboembolic complications was comparable for both substances [59]. Considering volume strain, possible allergic reactions, and the need for AB0-compatible transfusion of fresh plasma, according to current data PCC should be preferred to normalize INR levels as quickly as possible [1].

Furthermore, there are current results of two major multicenter cohort studies on the topic of antagonization in the acute phase. $A$ retrospective, pooled analysis of over $1500 \mathrm{VKA}-\mathrm{ICH}$ patients from 19 countries addressed the question of which agents can normalize INR most effectively [60]. The lowest rate of hematoma expansion, from lowest to highest, was achieved with a combination of PCC and FFP, PCC alone, and FFP alone (hazard ratio for hematoma expansion: $27.8 \%$ (reference), $37.3 \%, 45.6 \%$ ). Due to the retrospective design of this study, however, interpreting the study results for clinical application is critical, it may be more likely that the combined FFP + PCC approach could be viewed as an indicator for aggressive therapy in this treatment group. The previously mentioned German retrospective cohort study (RETRACE) of VKA-ICH patients was devoted to the question of which INR value should be targeted within which time window in order to minimize the rate of hematoma expansion. The rate of hematoma enlargement was significantly reduced, regardless of preparation administered (almost $90 \%$ with PCC alone or in combination), when the INR value achieved after correction was $<1.3$ (INR < 1.3: 26.9\% versus INR $\geq 1.3: 45.4 \%$; $p<0.001$ ) [10]. A more aggressive hemostatic treatment to INR values $<1$. 2 was not associated with any further drop in rate of hematoma expansion in this study. This positive effect of hemostatic treatment could be demonstrated up to approximately $4 \mathrm{~h}$ after hospital admission (INR < 1.3 within $4 \mathrm{~h}$ : $22.4 \%$ versus $42.2 \% ; p=0.02$ ) and was even more pronounced the earlier treatment was initiated and antagonization achieved (see - Fig. 1). In the adjusted analysis, effective (control INR < 1.3) and early (within $4 \mathrm{~h}$ after admission to hospital) anticoagulant reversal combined with a systolic blood pressure of $<160 \mathrm{mmHG}$ after $4 \mathrm{~h}$ was observed to reduce the hematoma expansion the most (OR 0.17 (95\% Cl: 0.11-0.33); $\mathrm{p}<0.001)$. Furthermore, in-hospital mortality of patients who were treated based on those targets was significantly reduced. Due to the negative results, safety concerns, and an increased rate of thromboembolic complications in the FAST trial, which was a phase III study on the efficacy of recombinant activated factor VII ( $\mathrm{rFVIIa)} \mathrm{in} \mathrm{ICH} \mathrm{patients,} \mathrm{the} \mathrm{administration} \mathrm{of} \mathrm{rFVIla} \mathrm{is} \mathrm{not}$ recommended at this time $[1,61]$. Existing studies produced neither proof of efficacy nor safety concerns for another substance, the antifibrinolytic tranexamic acid, which is currently being investigated in randomized trials (TICH: ISRCTN50867461 and STOP-AUST: NCT01702636). Due to its delayed effect, vitamin K is not appropriate for immediate coagulant reversal, but should be simultaneously replaced (10 mg i. v.) because otherwise INR can rise again due to PCC's extremely short half-life compared to conventional VKA $[1,62]$. After antagonization, INR checks should be conducted regularly and coagulation factors replaced again if needed [1].

- The goal for minimizing the risk of hematoma growth is immediate intravenous antagonization with PCC (see - Table 1 for dose) and vitamin K (10 mg i.v.) simultaneously.

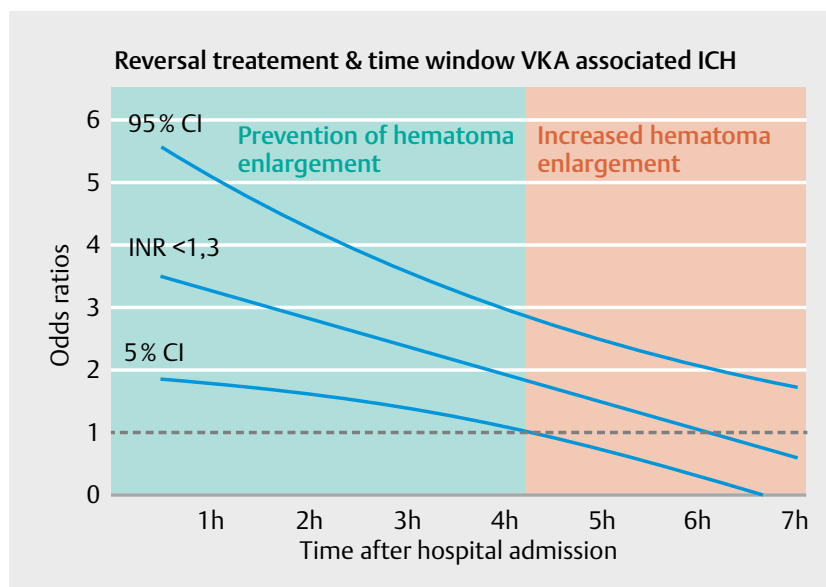

Reversal \& time window \& blood pressure management

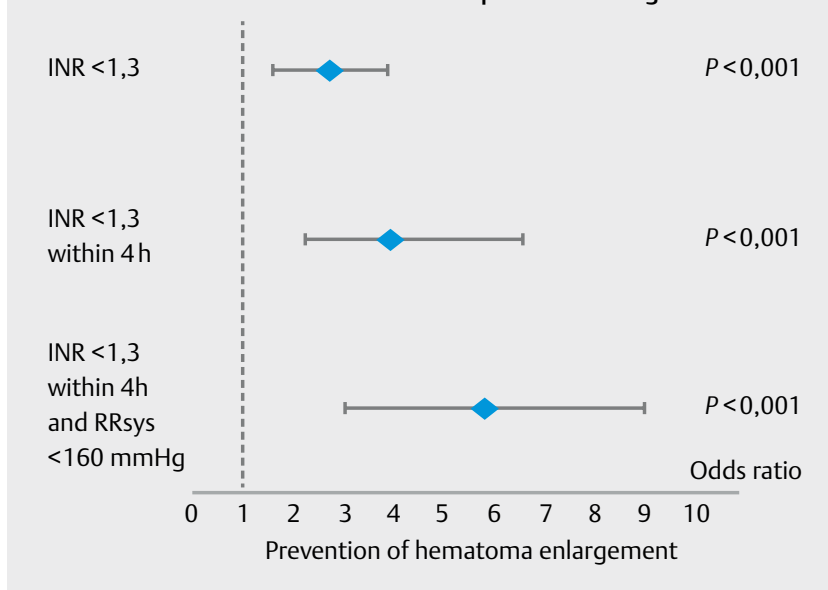

- Fig. 1 Acute treatment of VKA-ICH (RETRACE study, $n=853$ ) [10].

- Serial coagulation check should be obtained after reversal treatment.

- Antagonization to an INR target value at least less than 1.3, 1.2 possibly better.

- This target value $(I N R<1.3)$ should be achieved as fast as possible, at least $4 \mathrm{~h}$.

\section{Antagonization NOAC-ICH}

Based on the relatively short elimination half-life of NOACs (dabigatran 12-17 h, rivaroxaban 5-13 h, apixaban $12 \mathrm{~h}$, edoxaban 10-14 h) in comparison to VKA (marcumar 72-96 h), pausing the corresponding NOAC preparation could theoretically suffice in cases of NAOC-ICH, depending on the last ingestion and the concentration levels measured. However, this approach should be weighed very carefully because ICH is the most serious complication, mechanical hemostasis is impossible, and level measurement is unvalidated, time-consuming, and not available everywhere. Accordingly, hemostatic treatment should be immediately started for NOAC-ICHs as well.

Should anticoagulant reversal be necessary, either administering specific antidotes or replacing coagulation factors can be considered. At least for dabigatran, an effective and easy-to-administer antidote in the form of idarucizumab (Praxbind), a monoclonal antibody fragment, has been clinically available since the begin- 
ning of 2016. A published interim analysis and current conference data (Nov. 2016 American Heart Association Scientific Sessions; $n=494$ ) of the recently completed prospective, single-arm, open-label RE-VERSE AD study are available. The study demonstrated the safety and efficacy of this antibody to reverse the effects of dabigatran in patients with serious bleeding (Group $A, n=285$ ) or requiring an urgent surgical procedure (Group $B, n=196$ ) [63]. The primary endpoint of normalized coagulation test results (dilute thrombin time and ecarin clotting time) was achieved in all patients within $4 \mathrm{~h}$ by administering $5 \mathrm{~g}$ of idarucizumab (two boluses of $2.5 \mathrm{~g}$ i.v.). Dilute thrombin time was reduced within 10 to $30 \mathrm{~min}$ of administration and remained below the normal limit over $4 \mathrm{~h}$ for both coagulation times. However, it should be mentioned that at least on the basis of the data from the interim analysis, just under $25 \%$ of the patients had a normal dilute thrombin time at the time of inclusion in the study and therefore exhibited little or no effect of dabigatran. In patients requiring surgery, treating surgeons reported normal hemostasis in $92 \%$ of cases [63]. Accelerated approval was issued in November 2015, making idarucizumab available for daily clinical use in Germany to reverse the effects of dabigatran since the beginning of this year. An initial case report on its use for intracerebral hemorrhages in patients taking dabigatran appears promising; no hematoma expansion was observed in 2 patients after idarucizumab was administered [64]. For patients taking factor Xa inhibitors, a clinical trial is currently underway to investigate andexanet alfa, a recombinant factor $X$ a protein with no intrinsic catalytic activity. The ANNEXA-4 study examined the extent to which the effect of factor $X a$ inhibitors (rivaroxaban, apixaban, edoxaban and enoxaparin) could be reversed in patients with acute major bleeding [65]. Over 67 patients received a bolus of andexanet followed by a 2-h continuous infusion. After administration of the bolus, the median value for anti-factor Xa activity fell by $89 \%$ in patients taking rivaroxaban and $93 \%$ in patients taking apixaban, and $39 \%$ and $30 \%$ respectively four hours later. Twelve hours after the infusion, hemostasis was re-evaluated and $79 \%$ of patients with initially elevated anti-factor Xa levels (defined as $>75 \mathrm{ng} / \mathrm{ml}$ ) were assessed as good or better. For ICH patients, these results should be critically evaluated due to the very conservative definition of good coagulation, an increase in volume $<35 \%$ in ICH patients, and the long time interval ( $12 \mathrm{~h}$ ) between the end of infusion and evaluation of efficacy, which is approximately equal to the elimination half-life of antagonized NOAC derivates. An accelerated approval process based on the submitted data was not authorized by the FDA in the US [66], so andexanet alfa is currently not approved for clinical application outside of trials. Another antidote currently in development is ciraparantag (PER977), which unlike the above-mentioned preparations, is intended to bind and eliminate factor Xa inhibitors, dabigatran and heparin derivates through the formation of hydrogen bonds. Initial animal experiments were not very promising and approval is not expected any time soon [67].

Due to the lack of alternatives, the current European and international guidelines for ICHs that occur under treatment with factor Xa inhibitors recommend administering PCC or activated coagulation concentrates (FEIBA) at an initial dose of $50 \mathrm{lU} / \mathrm{kg}$ [68]. The recommendations are based mainly on experimental studies in which PCC was observed to prolong prothrombin time in healthy subjects taking rivaroxaban [69] and edoxaban reduced bleeding duration [70]. In the German-wide RASUNOA register, in which ischemic and hemorrhagic stroke patients under NOAC treatment were prospectively recorded, PCC was not observed to provide any benefit for NOAC-associated ICHs (hematoma expansion: 12/28 with PCC (43\%) versus 5/17 (29\%) without PCC; $p=0.53$ ) [50]. In a small case study of NOAC-associated ICHs $(n=5)$, no hematoma expansion occurred after administration of activated coagulation concentrates (FEIBA), however FEIBA infusion was begun within a median of $13 \mathrm{~h}$ (range $10-29 \mathrm{~h}$ ) from the last NOAC dose, so at this time effective anticoagulation was presumably no longer present depending on the medication [71]. If NOAC was taken within 2-4 $\mathrm{h}$ of the hemorrhage, activated charcoal can be administered to minimize the adsorption of the active substance $[72,73]$.

- The goal to minimize the risk of hemorrhage growth in the event of dabigatran-associated ICH is immediate intravenous antagonization with $5 \mathrm{~g}$ idarucizumab.

- The goal to minimize the risk of hemorrhage growth in the event of factor Xa inhibitor-associated ICH should be immediate intravenous antagonization with at least $50 \mathrm{lU} / \mathrm{kg}$ PCC or FEIBA (no evidence).

- The administration of active charcoal may be considered if time of ingestion is known to be early (within $4 \mathrm{~h}$ after onset of symptoms).

\section{Acute Treatment Starting Day 2}

Patients with ICH should always be treated in specialized centers with a high annual throughput. This recommendation concurs with the current guidelines and is based on valid evidence. An American prospective study of ICH patients showed increased in-hospital mortality when patients were treated in a general ICU compared with a specialized neuro ICU $(\mathrm{OR}=3.4 ; \mathrm{Cl} 1.6-7.6)$, with high patient volume and size of ICU favorably affecting outcome [74]. A more recent study of $10000 \mathrm{ICU}$ patients treated in either a neuro or general ICU confirmed lower in-hospital mortality of ICH patients treated in the specialized neurological ICU [75]. The effects were also similar for less acute ICH patients treated in a stroke unit. A meta-analysis that assessed 13 randomized studies likewise evidenced the cited noticeable benefits [76]. The main reasons for this association are presumably the expertise of the treatment team and the dedicated monitoring (clinical, multimodal, and/or invasive) to enable early detection of threatening secondary complications and a swift response [77]. Furthermore, such specialized neuro intensive care represents a high logistical, operational, and personnel expense that these specialized centers can carry. There is hardly any reliable data available explicitly for OAC-ICH patients on the treatment aspects described in the following. The attempt was therefore made to place the treatment procedures in the context of the specific characteristics of $\mathrm{OAC}-\mathrm{ICH}$ and derive a possible relevance for OAC-ICH patients.

\section{Hematoma evacuation (craniectomy, minimally invasive procedure)}

The STICH 1 study was the first major randomized study that investigated hematoma evacuation surgery in over 1000 patients with supratentorial ICH. It compared early hematoma evacuation (diagnosis within $72 \mathrm{~h}$ after onset of symptoms) with conservative treat- 
ment. The surgical approach appeared to have a small advantage for patients with superficial, atypical ICH [78], which was confirmed in meta-analyses of existing RCTs on surgical treatment after ICH. The STICH 2 study followed, which investigated a neurosurgical approach in patients with lobar ICH (volume between 10-100 ml) and no intraventricular hemorrhage, located near the cortex $(<1 \mathrm{~cm})$, and accessible for early surgical intervention [79]. A total of 601 patients were randomized and of those surgically treated nearly all patients (98\%) underwent an open craniotomy with hematoma evacuation. There were no relevant differences with respect to the functional endpoint of the study between the compared procedures in the intention-to-treat analysis (OR 0.86 (0.62-1.20); $p=0.36$ ). The validity of the study is undermined by the high number of patients ( $21 \%$ ) in the conservative treatment arm who nonetheless were later treated surgically because their clinical condition worsened but who, for the purposes of the primary endpoint analysis, were categorized in the conservative-treatment group. Therefore the slight advantage of surgery - at least for mortality at 6 months - may actually be greater. Minimally invasive strategies (catheter-based or endoscopic) are being increasingly studied in light of the sobering results of open surgical procedures. Various meta-analyses based on predominantly smaller studies suggest, at least for the minimally invasive approach, advantages in terms of reduced mortality and morbidity compared to other surgical as well as conservative procedures. The MISTIE II study was a larger randomized study that investigated, in 118 patients with a supratentorial ICH, a combined approach of a minimally invasive catheter application and hematoma lysis with rt-PA compared to conservative treatment. Ultimately the intervention significantly reduced the size of the hematoma and edema without influencing the functional endpoints $[80,81]$. The follow-up study (MISTIE III) is currently recruiting and is designed to investigate the efficacy and safety of the procedure adequately (planned inclusion of 500 patients, until end of 2018) (NCT01827046). Other technical innovations using various endoscopic systems (e. g., the INVEST study) are currently under investigation. Another albeit more invasive approach - derived from operative RCTs for malignant middle cerebral artery infarction - is decompressive hemicraniectomy in patients with basal ganglia ICH (SWITCH study), which randomly compares it to maximally conservative treatment (NCT 02258919),

It is somewhat difficult to derive aspects relevant to $\mathrm{OAC}-\mathrm{ICH}$ from the surgical approach, however subjective reports indicate that an OAC-ICH may be easier to evacuate because of the altered coagulation, but data to support this view is lacking. In general, OAC-ICH is justifiably excluded from surgical trials because altered hemostasis could theoretically persist in spite of systemic antagonization in the hematoma cavity. Hemicraniectomy also harbors possible increased risks because the tamponade effect from cranial bone is removed, and the hematoma encounters less resistance to stopping, prolonged such as in $\mathrm{OAC}-\mathrm{ICH}$.

\section{External ventricle drainage, intraventricular fibrinoly- sis and lumbar drainage}

Intraventricular hemorrhage (IVH) is a known predictor of poor outcome and complicates the treatment of $\mathrm{ICH}$ in approximately $50 \%$ of patients. IVH is an immediate, life-threatening complication that due to the obstruction of the ventricular system - especially the third and fourth ventricles - can cause an acute hydrocephalus ultimately leading to herniation [82]. The method of choice for treating the acute rise in intracranial pressure and preventing herniation is placement of an external ventricular drain (EVD). In the ongoing course of treatment, procedures such as intraventricular lysis, endoscopic techniques, and overlapping lumbar drainage offer options to clear intraventricular blood more quickly, and correlated positively with clinical endpoints in various observational and prospective pilot studies [83-85]. According to the latest data, intraventricular lysis via rt-PA or urokinase via EVD is injected into the ventricle system but only after CT has confirmed the size of the hematoma is stable. This is performed via one or two EVDs with e. $g$., $1 \mathrm{mg}$ rt-PA, every $8-12 \mathrm{~h}$ until the third and fourth ventricle are clear of blood and communication between the ventricular and subarachnoid cerebral spinal fluid space is re-established [46]. This approach was investigated on a large scale for the first time in the CLEAR-IVH study, starting in 2003. The promising results led to the CLEAR III study, which is complete but as yet unpublished. Although the results with respect to the primary endpoint (improved functional status at 6 months) are negative, there is a significant association with an improved outcome especially in patients with pronounced IVH (IVH volume > $20 \mathrm{ml})(\mathrm{OR}: 1.84, \mathrm{p}=0.046)$ at an existing effect size of just under $10 \%$. But the treatment was associated with a $10 \%$ reduction in mortality as a secondary endpoint (data presented at ISC 2016). Another promising approach is overlapping lumbar drainage. Theoretically, it should re-establish the physiological circulation of CSF as quickly as possible and remove intraventricular and subarachnoid blood from the intracranial compartment. It is hoped that it will prevent exacerbation from blood and its breakdown products - the strong inflammation mediators. Note that lumbar drainage is a simple bedside procedure that can be performed during the subacute course. Before placing the lumbar drain, however, communication between the internal and external CSF system must be confirmed as re-established, otherwise herniation could theoretically occur. Previous pilot and observational studies showed that the combination of intraventricular fibrinolysis and lumbar drainage might be associated with reduced permanent shunt dependency and an improved outcome [84, 85]. A smaller, very recent randomized study, including a meta-analysis based on individual patient data, confirmed in just under 100 patients the safety of this procedure and its efficacy in avoiding permanent shunts [86]. Endoscopic aspiration of the intraventricular blood represents another approach, with various techniques already having been described. A small randomized study ( $n=48$ ) likewise resulted in a significant reduction in shunt rate [87].

Although the studies cited largely excluded OAC-ICH patients, there are several interesting implications considering the characteristics of an ICH while under anticoagulation treatment. As already mentioned, $\mathrm{OAC}-\mathrm{ICH}$ is associated with a more frequent and greater extent of IVH. Pathophysiological considerations suggest that IVF with or without lumbar drainage is not only transferable but could also be more relevant than in primary ICH because more than half of patients are affected. Furthermore, CLEAR III demonstrated a positive effect on functional outcome particularly in cases of pronounced ventricular intrusion, which is much more frequent in $\mathrm{OAC}-\mathrm{ICH}$. Note, however, that these considerations are merely 
theoretical and there is no evidence to date regarding safety in OAC-ICHs.

\section{Treatment of perihemorrhagic edema}

After $\mathrm{ICH}$, perihemorrhagic edema frequently develops that is associated with premature neurological deterioration and is therefore another negative prognostic parameter $[88,89]$. The temporal course can be divided into two phases - acute $(0-24 \mathrm{~h})$ and late phase (10-14 days) with maximum edema $[90,91]$. In the acute phase, extravasation of pro-osmotic substances from the blood clot (proteins, electrolytes) occurs, which can cause premature vasogenic edema [92]. Over time (the next several hours to days), the activation of the coagulation cascade begins increased thrombin production with an osmotic effect as well as the release of inflammation mediators through the breakdown of erythrocytes and hemoglobin. This might be called a mixed vasogenic and cytotoxic edema $[91,92]$. The underlying mechanism of damage is the volume effect of the edema, which can lead to intracranial hypertension with consecutive cerebral hypoperfusion (local to global) and secondary brain damage $[92,93]$. A wide variety of substances (hypertonic sodium chloride, mannitol, steroids, chelating agents) and interventions (hypothermia, decompressive craniectomy) are used to treat perihemorrhagic edema, but to date there is no valid evidence for definitively recommending their use $[1,12]$. A current subanalysis of the INTERACT 2 data identified associations with an improved outcome for mannitol, at least for larger bleeds (>15 ml) [94]. Promising data has emerged from cohort and pilot studies for hypertonic sodium chloride infusions, among others, deferoxamine (recently published), and mild, prolonged hypothermia $[88,95,96]$. A current review identified 18 studies on the influence of hypothermia on edema after ICH. Treatment using hypothermia was significantly associated with reduced edema development and advantages for clinical endpoints. Results from randomized trials are currently unknown (ISRCTN28699995; NCT01607151). Another subanalysis of the INTERACT 2 data examined temperature regulation and edema development and established a linear relationship between edema and ambient temperature, i. e., a higher ambient temperature increased the volume of perihemorrhagic edema [97]. The effect of a decompressive craniectomy was also investigated and did show progressive and prolonged edema after surgery, although the clinical outcome for those patients appeared better [98].

For OAC-ICHs in those receiving vitamin $\mathrm{K}$ antagonists, experimental data suggest a possible reduction in edema because thrombin production appears to be less pronounced, although there are very few clinical trials. Hence no OAC-ICH-specific treatment approach can be inferred, but early initiation of hypothermia should possibly be avoided because it can alter coagulation. This stands in contrast to study results in primary ICH patients that showed hypothermia to positively affect perihemorrhagic edema only when initiated early ( $<72 \mathrm{~h}$ after onset of symptoms) [96].

\section{Thrombosis and thromboembolism prophylaxis}

Compared to ischemic stroke patients, ICH patients suffer much greater functional impairment, which is associated with a higher degree of immobilization and hence a greater risk of thrombosis. Early mobilization of stroke patients is therefore viewed as the most effective way to prevent deep vein thrombosis (DVT) and pulmonary emboli (PE), but ICH patients can be adequately mobilized only to a limited extent. To date, only few studies have investigated early thrombosis prophylaxis in $\mathrm{ICH}$ patients. A related meta-analysis indicated that prophylaxis with heparinoids (unfractionated, low-molecular-weight) may be associated with an increased risk of symptomatic intracerebral hemorrhage ( $8.0 \%$ versus $4.0 \%$; RR, 1.42 (95\% Cl: 0.57-3.53; $p=0.45$ ), although it does provide significant protection against pulmonary emboli ( $1.7 \%$ versus $2.9 \%$; RR 0.37 (95\% Cl: 0.17-0.80); $p=0.01$ ) [99]. Thrombosis prophylaxis was not demonstrated to have an effect on mortality. Another subanalysis of the INTERACT trial population looked at the impact of administering prophylactic heparin; a propensity-score-matched analysis indicated a significantly higher rate of disability in heparin-treated patients, although it is unclear whether the statistically rigorous adjustment procedure adequately corrected the indication bias [100]. For stroke patients in general, the prospective CLOTS trials (Clots in Legs Or sTockings after Stroke), which investigated the effectiveness and safety of intermittent, pneumatic compression, produced the best evidence on the topic of thrombosis prophylaxis and reported a prevalence of symptomatic deep vein thromboses of less than $5 \%$ and lung emboli of less than $2 \%$ [101]. These trial data did not support the use of compression stockings in stroke patients due to undesirable complications (skin necrosis, ulcers) [101]. The results of the subsequent CLOTS 3 trial showed this intervention to have a significantly lower risk of deep vein thromboses and possibly higher survival rate in $\mathrm{ICH}$ patients, although skin lesions were more frequent [102].

Compared to general stroke patients, OAC-ICH patients per se have a higher risk of thromboembolic complications. These patients are therefore in a special risk group in which the increased risk of hemorrhage must be weighed against the risk of thromboembolism and the treatment decision (early anticoagulation) considered on an individual basis. Based on the current state of the data, in general the best-possible, non-drug prophylactic treatment should be pursued because there is no valid evidence that early anticoagulation prevents a deep vein thrombosis or systemic thromboembolism in ICH patients. In particular, patients with mechanical heart valves are another subgroup at greater risk of thromboembolism. Safety considerations should therefore prevail in treating these patients - and if justifiable - early anticoagulation be avoided. Older data report a manageable risk of ischemia of less than $3 \%$ within the first 30 days after discontinuing OAK.

\section{Practical Summary}

- OAC-ICH is the most serious form of hemorrhagic stroke and its incidence is expected to grow.

- One-year mortality is over $50 \%$ and fewer than $30 \%$ of patients recover functional independence (modified Rankin scale 0-3).

- The main treatment goal in the acute phase should be minimizing hematoma growth.

- In all cases of OAC-ICH, immediate, intensified measures to lower blood pressure should be taken (target systolic blood pressure $140 \mathrm{mmHg}$ ). 
- In cases of VKA-ICH, immediate antagonization (at least within $4 \mathrm{~h}$ ) to INR values less than 1.3 or 1.2 using PCC and vitamin $\mathrm{K}$ should be pursued.

- For dabigatran-associated ICH, 5 mg of idarucizumab should be administered immediately for antagonization.

- There is no evidence in the case of factor Xa inhibitor-associated ICH, and antagonization using PCC or FEIBA should be considered as a substitute.

- In the sub-acute phase (as of day 2), specific ICH therapies are employed, but an individual risk-benefit analysis is of utmost importance with respect to increased risk of hemorrhage and thromboembolism (hematoma evacuation, edema therapy, thrombosis prophylaxis).

- Intraventricular fibrinolysis with overlapping lumbar drainage, if needed, could theoretically be clinically relevant because of the increased and greater extent of IVH that is characteristic in OAC-ICHs.

\section{Conflict of Interest}

No conflict of interest has been declared by the authors.

\section{References}

[1] Hemphill JC 3rd, Greenberg SM, Anderson CS et al. Guidelines for the management of spontaneous intracerebral hemorrhage: A guideline for healthcare professionals from the American Heart Association/ American Stroke Association. Stroke 2015; 46: 2032-2060

[2] Qureshi Al, Mendelow AD, Hanley DF. Intracerebral haemorrhage. Lancet 2009; 373: 1632-1644

[3] Sacco S, Marini C, Toni D et al. Incidence and 10-year survival of intracerebral hemorrhage in a population-based registry. Stroke 2009; 40: 394-399

[4] Biffi A, Battey TW, Ayres AM et al. Warfarin-related intraventricular hemorrhage: imaging and outcome. Neurology 2011; 77: 1840-1846

[5] Flaherty ML, Tao H, Haverbusch M et al. Warfarin use leads to larger intracerebral hematomas. Neurology 2008; 71: 1084-1089

[6] Flibotte J], Hagan N, O’Donnell J et al. Warfarin, hematoma expansion, and outcome of intracerebral hemorrhage. Neurology 2004; 63: 1059-1064

[7] Rosand J, Eckman MH, Knudsen KA et al. The effect of warfarin and intensity of anticoagulation on outcome of intracerebral hemorrhage. Arch Intern Med 2004; 164: 880-884

[8] Frontera JA, Lewin JJ 3rd, Rabinstein AA et al. Guideline for reversal of antithrombotics in intracranial hemorrhage: A statement for healthcare professionals from the Neurocritical Care Society and Society of Critical Care Medicine. Neurocrit Care 2016; 24: 6-46

[9] Gage BF, Waterman AD, Shannon W et al. Validation of clinical classification schemes for predicting stroke: Results from the National Registry of Atrial Fibrillation. JAMA 2001; 285: 2864-2870

[10] Kuramatsu JB, Gerner ST, Schellinger PD et al. Anticoagulant reversal, blood pressure levels, and anticoagulant resumption in patients with anticoagulation-related intracerebral hemorrhage. JAMA 2015; 313 : 824-836

[11] Pisters R, Lane DA, Nieuwlaat R et al. A novel user-friendly score (HAS-BLED) to assess 1-year risk of major bleeding in patients with atrial fibrillation: the Euro Heart Survey. Chest 2010; 138: 1093-1100
[12] Steiner T, Al-Shahi Salman R, Beer R et al. European Stroke Organisation (ESO) guidelines for the management of spontaneous intracerebral hemorrhage. Int J Stroke 2014; 9: 840-855

[13] Heidbuchel H, Verhamme P, Alings M et al. Updated European Heart Rhythm Association practical guide on the use of non-vitamin-K antagonist anticoagulants in patients with non-valvular atrial fibrillation: Executive summary. Eur Heart ] 2016, doi:10.1093/ eurheartj/ehw058

[14] Krishnamurthi RV, Feigin VL, Forouzanfar MH et al. Global and regional burden of first-ever ischaemic and haemorrhagic stroke during 1990-2010: Findings from the Global Burden of Disease Study 2010. Lancet Glob Health 2013; 1: e259-e281

[15] Nielsen PB, Larsen TB, Skjoth F et al. Restarting anticoagulant treatment after intracranial hemorrhage in patients with atrial fibrillation and the impact on recurrent stroke, mortality, and bleeding: A nationwide cohort study. Circulation 2015; 132: 517-525

[16] Schols AM, Schreuder FH, van Raak EP et al. Incidence of oral anticoagulant-associated intracerebral hemorrhage in the Netherlands. Stroke 2014; 45: 268-270

[17] Hart RG, Boop BS, Anderson DC. Oral anticoagulants and intracranial hemorrhage. Facts and hypotheses. Stroke 1995; 26: 1471-1477

[18] Flaherty ML, Kissela B, Woo D et al. The increasing incidence of anticoagulant-associated intracerebral hemorrhage. Neurology 2007; 68: $116-121$

[19] Rosand J, Muzikansky A, Kumar A et al. Spatial clustering of hemorrhages in probable cerebral amyloid angiopathy. Ann Neurol 2005; 58 : 459-462

[20] Greenberg SM, Al-Shahi Salman R, Biessels G] et al. Outcome markers for clinical trials in cerebral amyloid angiopathy. Lancet Neurol 2014; 13: $419-428$

[21] Patel N], Deshmukh A, Pant S et al. Contemporary trends of hospitalization for atrial fibrillation in the United States, 2000 through 2010: Implications for healthcare planning. Circulation 2014; 129: 2371-2379

[22] Caldeira D, Rodrigues FB, Barra M et al. Non-vitamin K antagonist oral anticoagulants and major bleeding-related fatality in patients with atrial fibrillation and venous thromboembolism: A systematic review and meta-analysis. Heart 2015; 101: 1204-1211

[23] Hylek EM, Evans-Molina C, Shea C et al. Major hemorrhage and tolerability of warfarin in the first year of therapy among elderly patients with atrial fibrillation. Circulation 2007; 115: 2689-2696

[24] Skaistis J, Tagami T. Risk of fatal bleeding in episodes of major bleeding with new oral anticoagulants and vitamin $\mathrm{K}$ antagonists: A systematic review and meta-analysis. PLoS One 2015; 10: e0137444

[25] Claassen DO, Kazemi N, Zubkov AY et al. Restarting anticoagulation therapy after warfarin-associated intracerebral hemorrhage. Arch Neurol 2008; 65: 1313-1318

[26] Molina CA, Selim MH. The dilemma of resuming anticoagulation after intracranial hemorrhage: Little evidence facing big fears. Stroke 2011; 42: 3665-3666

[27] Biffi A, Anderson CD, Battey TW et al. Association between blood pressure control and risk of recurrent intracerebral hemorrhage. JAMA 2015; 314: 904-912

[28] AlKherayf F, Xu Y, Gandara E et al. Timing of vitamin K antagonist re-initiation following intracranial hemorrhage in mechanical heart valves: Systematic review and meta-analysis. Thromb Res 2016; 144 : 152-157

[29] Apostolakis S, Lane DA, Guo Y et al. Performance of the HEMORR(2) HAGES, ATRIA, and HAS-BLED bleeding risk-prediction scores in patients with atrial fibrillation undergoing anticoagulation: the AMADEUS (evaluating the use of SR34006 compared to warfarin or acenocoumarol in patients with atrial fibrillation) study. J Am Coll Cardiol 2012; 60: 861-867 
[30] Apostolakis S, Lane DA, Buller $\mathrm{H}$ et al. Comparison of the CHADS2, CHA2DS2-VASC and HAS-BLED scores for the prediction of clinically relevant bleeding in anticoagulated patients with atrial fibrillation: The AMADEUS trial. Thromb Haemost 2013; 110: 1074-1079

[31] Hylek EM, Go AS, Chang Y et al. Effect of intensity of oral anticoagulation on stroke severity and mortality in atrial fibrillation. N Engl J Med 2003; 349: 1019-1026

[32] Stein M, Misselwitz B, Hamann GF et al. Intracerebral hemorrhage in the very old: Future demographic trends of an aging population. Stroke 2012; 43: 1126-1128

[33] Hankey G], Stevens SR, Piccini JP et al. Intracranial hemorrhage among patients with atrial fibrillation anticoagulated with warfarin or rivaroxaban: The rivaroxaban once daily, oral, direct factor Xa inhibition compared with vitamin $\mathrm{K}$ antagonism for prevention of stroke and embolism trial in atrial fibrillation. Stroke 2014; 45: 1304-1312

[34] Eikelboom JW, Wallentin L, Connolly SJ et al. Risk of bleeding with 2 doses of dabigatran compared with warfarin in older and younger patients with atrial fibrillation: An analysis of the randomized evaluation of long-term anticoagulant therapy (RE-LY) trial. Circulation 2011; 123: 2363-2372

[35] Verheugt FW, Granger CB. Oral anticoagulants for stroke prevention in atrial fibrillation: Current status, special situations, and unmet needs. Lancet 2015; 386: 303-310

[36] Smith EE, Eichler F. Cerebral amyloid angiopathy and lobar intracerebral hemorrhage. Arch Neurol 2006; 63: 148-151

[37] Schneider JA. The cerebral cortex in cerebral amyloid angiopathy. Lancet Neurol 2016; 15: 778-779

[38] Lovelock CE, Cordonnier C, Naka H et al. Antithrombotic drug use, cerebral microbleeds, and intracerebral hemorrhage: A systematic review of published and unpublished studies. Stroke 2010; 41: $1222-1228$

[39] van Etten ES, Auriel E, Haley KE et al. Incidence of symptomatic hemorrhage in patients with lobar microbleeds. Stroke 2014; 45: 2280-2285

[40] O'Donnell HC, Rosand J, Knudsen KA et al. Apolipoprotein E genotype and the risk of recurrent lobar intracerebral hemorrhage. $N$ Engl J Med 2000; 342: 240-245

[41] Hemphill JC 3rd, Farrant M, Neill TA Jr.. Prospective validation of the ICH Score for 12-month functional outcome. Neurology 2009; 73 : 1088-1094

[42] Rost NS, Smith EE, Chang Y et al. Prediction of functional outcome in patients with primary intracerebral hemorrhage: the FUNC score. Stroke 2008; 39: 2304-2309

[43] Hemphill JC 3rd, Bonovich DC, Besmertis L et al. The ICH score: A simple, reliable grading scale for intracerebral hemorrhage. Stroke 2001; 32: 891-897

[44] Parry-Jones AR, Abid KA, Di Napoli M et al. Accuracy and clinical usefulness of intracerebral hemorrhage grading scores: A direct comparison in a UK population. Stroke 2013; 44: 1840-1845

[45] Koennecke HC, Belz W, Berfelde D et al. Factors influencing in-hospital mortality and morbidity in patients treated on a stroke unit. Neurology 2011; 77: 965-972

[46] Ziai WC, Tuhrim S, Lane K et al. A multicenter, randomized, double-blinded, placebo-controlled phase III study of Clot Lysis Evaluation of Accelerated Resolution of Intraventricular Hemorrhage (CLEAR III). Int J Stroke 2014; 9: 536-542

[47] Broderick JP, Brott TG, Duldner JE et al. Volume of intracerebral hemorrhage. A powerful and easy-to-use predictor of 30-day mortality. Stroke 1993; 24: 987-993

[48] Wang X, Arima H, Al-Shahi Salman R et al. Clinical prediction algorithm (BRAIN) to determine risk of hematoma growth in acute intracerebral hemorrhage. Stroke 2015; 46: 376-381
[49] Brouwers HB, Chang Y, Falcone G] et al. Predicting hematoma expansion after primary intracerebral hemorrhage. JAMA Neurol 2014; 71: 158-164

[50] Purrucker JC, Haas K, Rizos T et al. Early clinical and radiological course, management, and outcome of intracerebral hemorrhage related to new oral anticoagulants. JAMA Neurol 2016; 73: 169-177

[51] Anderson CS, Huang Y, Wang JG et al. Intensive blood pressure reduction in acute cerebral haemorrhage trial (INTERACT): $\mathrm{A}$ randomised pilot trial. The Lancet Neurology 2008; 7: 391-399

[52] Qureshi Al, Palesch YY, Martin R et al. Effect of systolic blood pressure reduction on hematoma expansion, perihematomal edema, and 3-month outcome among patients with intracerebral hemorrhage: results from the antihypertensive treatment of acute cerebral hemorrhage study. Arch Neurol 2010; 67: 570-576

[53] Butcher KS, Jeerakathil T, Hill M et al. The intracerebral hemorrhage acutely decreasing arterial pressure trial. Stroke 2013; 44: 620-626

[54] Anderson CS, Heeley E, Huang Y et al. Rapid blood-pressure lowering in patients with acute intracerebral hemorrhage. N Engl J Med 2013; 368: $2355-2365$

[55] Qureshi Al, Palesch YY, Barsan WG et al. Intensive blood-pressure lowering in patients with acute cerebral hemorrhage. N Engl J Med 2016; 375: 1033-1043

[56] (CHMP), E.M.A.-C.f.M.P.f.H.U.. Nicardipine-containing medicinal products for intravenous use. 2013

[57] Sarode R, Milling T] Jr., Refaai MA et al. Efficacy and safety of a 4-factor prothrombin complex concentrate in patients on vitamin $\mathrm{K}$ antagonists presenting with major bleeding: a randomized, plasma-controlled, phase IIIb study. Circulation 2013; 128: 1234-1243

[58] Goldstein JN, Refaai MA, Milling T] Jr. et al. Four-factor prothrombin complex concentrate versus plasma for rapid vitamin $\mathrm{K}$ antagonist reversal in patients needing urgent surgical or invasive interventions: a phase 3b, open-label, non-inferiority, randomised trial. Lancet 2015; 385: 2077-2087

[59] Steiner T, Poli S, Griebe M et al. Fresh frozen plasma versus prothrombin complex concentrate in patients with intracranial haemorrhage related to vitamin $\mathrm{K}$ antagonists (INCH): a randomised trial. Lancet Neurol 2016; 15: 566-573

[60] Parry-Jones AR, Di Napoli M, Goldstein JN et al. Reversal strategies for vitamin $\mathrm{K}$ antagonists in acute intracerebral hemorrhage. Ann Neurol 2015; 78: 54-62

[61] Mayer SA, Brun NC, Begtrup K et al. Efficacy and Safety of Recombinant Activated Factor VII for Acute Intracerebral Hemorrhage. N Engl J Med 2008; 358: 2127-2137

[62] Raj G, Kumar R, McKinney WP. Time course of reversal of anticoagulant effect of warfarin by intravenous and subcutaneous phytonadione. Arch Intern Med 1999; 159: 2721-2724

[63] Pollack CV Jr., Reilly PA, Eikelboom J et al. Idarucizumab for dabigatran reversal. N Engl J Med 2015; 373: 511-520

[64] Held V, Eisele P, Eschenfelder CC et al. Idarucizumab as antidote to intracerebral hemorrhage under treatment with dabigatran. Case Rep Neurol 2016; 8: 224-228

[65] Connolly SJ, Milling TJ Jr., Eikelboom JW et al. Andexanet alfa for acute major bleeding associated with factor Xa inhibitors. N Engl J Med 2016; 375: 1131-1341

[66] Portola Pharmaceuticals, I. 2016; Portola Pharmaceuticals receives complete response letter from FDA for Biologics License Application for AndexXa ${ }^{\mathrm{TM}}$ (andexanet alfa).

[67] Ansell JE, Bakhru SH, Laulicht BE et al. Use of PER977 to reverse the anticoagulant effect of edoxaban. N Engl J Med 2014; 371: 2141-2142

[68] Heidbuchel H, Verhamme P, Alings M et al. Updated European Heart Rhythm Association Practical Guide on the use of non-vitamin K antagonist anticoagulants in patients with non-valvular atrial fibrillation. Europace 2015; 17: 1467-1507 
[69] Eerenberg ES, Kamphuisen PW, Sijpkens MK et al. Reversal of rivaroxaban and dabigatran by prothrombin complex concentrate: a randomized, placebo-controlled, crossover study in healthy subjects. Circulation 2011; 124: 1573-1579

[70] Zahir H, Brown KS, Vandell AG et al. Edoxaban effects on bleeding following punch biopsy and reversal by a 4 -factor prothrombin complex concentrate. Circulation 2015; 131: 82-90

[71] Dibu JR, Weimer JM, Ahrens $C$ et al. The role of FEIBA in reversing novel oral anticoagulants in intracerebral hemorrhage. Neurocrit Care 2016; 24: 413-419

[72] van Ryn J, Sieger P, Kink-Eiband M et al. Adsorption of dabigatran etexilate in water or dabigatran in pooled human plasma by activated charcoal in vitro. Blood 2009; 114: 440-440

[73] Wang X, Mondal S, Wang J et al. Effect of activated charcoal on apixaban pharmacokinetics in healthy subjects. Am J Cardiovasc Drugs 2014; 14: 147-154

[74] Diringer MN, Edwards DF. Admission to a neurologic/neurosurgical intensive care unit is associated with reduced mortality rate after intracerebral hemorrhage. Crit Care Med 2001; 29: 635-640

[75] Damian MS, Ben-Shlomo Y, Howard R et al. The effect of secular trends and specialist neurocritical care on mortality for patients with intracerebral haemorrhage, myasthenia gravis and Guillain-Barre syndrome admitted to critical care: an analysis of the Intensive Care National Audit \& Research Centre (ICNARC) national United Kingdom database. Intensive Care Med 2013; 39: 1405-1412

[76] Langhorne P, Fearon P, Ronning OM et al. Stroke unit care benefits patients with intracerebral hemorrhage: systematic review and meta-analysis. Stroke 2013; 44: 3044-3049

[77] Evans A, Perez I, Harraf F et al. Can differences in management processes explain different outcomes between stroke unit and stroke-team care? Lancet 2001; 358: 1586-1592

[78] Mendelow AD, Gregson BA, Fernandes HM et al. Early surgery versus initial conservative treatment in patients with spontaneous supratentorial intracerebral haematomas in the International Surgical Trial in Intracerebral Haemorrhage (STICH): a randomised trial. Lancet 2005; 365: 387-397

[79] Mendelow AD, Gregson BA, Rowan EN et al. Early surgery versus initial conservative treatment in patients with spontaneous supratentorial lobar intracerebral haematomas (STICH II): A randomised trial. Lancet 2013; 382: 397-408

[80] Mould WA, Carhuapoma JR, Muschelli ] et al. Minimally invasive surgery plus recombinant tissue-type plasminogen activator for intracerebral hemorrhage evacuation decreases perihematomal edema. Stroke 2013; 44: 627-634

[81] Hanley DF, Thompson RE, Muschelli J et al. Safety and efficacy of minimally invasive surgery plus alteplase in intracerebral haemorrhage evacuation (MISTIE): A randomised, controlled, open-label, phase 2 trial. Lancet Neurol 2016; 15: 1228-1237

[82] Staykov D, Schwab S. Clearing bloody cerebrospinal fluid: Clot lysis, neuroendoscopy and lumbar drainage. Curr Opin Crit Care 2013; 19 : 92-100

[83] Hanley DF. Intraventricular hemorrhage: severity factor and treatment target in spontaneous intracerebral hemorrhage. Stroke 2009; 40: 1533-1538

[84] Staykov D, Huttner HB, Struffert T et al. Intraventricular fibrinolysis and lumbar drainage for ventricular hemorrhage. Stroke 2009; 40: $3275-3280$

[85] Huttner HB, Nagel S, Tognoni E et al. Intracerebral hemorrhage with severe ventricular involvement: Lumbar drainage for communicating hydrocephalus. Stroke 2007; 38: 183-187
[86] Staykov D, Kuramatsu JB, Bardutzky J et al. Efficacy and safety of combined intraventricular fibrinolysis with lumbar drainage for prevention of permanent shunt dependency after intracerebral hemorrhage with severe ventricular involvement: A randomized trial and individual patient data meta-analysis. Ann Neurol 2016; 81: 93-103

[87] Chen CC, Liu CL, Tung YN et al. Endoscopic surgery for intraventricular hemorrhage (IVH) caused by thalamic hemorrhage: Comparisons of endoscopic surgery and external ventricular drainage (EVD) surgery. World Neurosurg 2011; 75: 264-268

[88] Volbers B, Willfarth W, Kuramatsu JB et al. Impact of perihemorrhagic edema on short-term outcome after intracerebral hemorrhage. Neurocrit Care 2016; 24: 404-412

[89] Murthy SB, Moradiya Y, Dawson J et al. Perihematomal edema and functional outcomes in intracerebral hemorrhage: Influence of hematoma volume and location. Stroke 2015; 46: 3088-3092

[90] Gebel JM Jr., Jauch EC, Brott TG et al. Natural history of perihematomal edema in patients with hyperacute spontaneous intracerebral hemorrhage. Stroke 2002; 33: 2631-2635

[91] Kuramatsu JB, Huttner HB, Schwab S. Advances in the management of intracerebral hemorrhage. J Neural Transm (Vienna) 2013; 120 (Suppl 1): S35-S41

[92] Xi G, Keep RF, Hoff JT. Mechanisms of brain injury after intracerebral haemorrhage. Lancet Neurol 2006; 5: 53-63

[93] Rincon F, Mayer SA. Novel therapies for intracerebral hemorrhage. Curr Opin Crit Care 2004; 10: 94-100

[94] Wang X, Arima H, Yang J et al. Mannitol and outcome in intracerebral hemorrhage: propensity score and multivariable intensive blood pressure reduction in acute cerebral hemorrhage trial 2 results. Stroke 2015; 46: 2762-2767

[95] Yu Y, Zhao W, Zhu C et al. The clinical effect of deferoxamine mesylate on edema after intracerebral hemorrhage. PLoS One 2015; 10: e0122371

[96] Volbers B, Herrmann S, Willfarth W et al. Impact of hypothermia initiation and duration on perihemorrhagic edema evolution after intracerebral emorrhage. Stroke 2016; 47: 2249-2255

[97] Zheng D, Arima H, Heeley E et al. Ambient temperature and volume of perihematomal edema in acute intracerebral haemorrhage: the INTERACT1 study. Int J Stroke 2015; 10: 25-27

[98] Fung C, Murek M, Klinger-Gratz PP et al. Effect of decompressive craniectomy on perihematomal edema in patients with intracerebral hemorrhage. PLoS One 2016; 11: e0149169

[99] Geeganage CM, Sprigg N, Bath MW et al. Balance of symptomatic pulmonary embolism and symptomatic intracerebral hemorrhage with low-dose anticoagulation in recent ischemic stroke: A systematic review and meta-analysis of randomized controlled trials. J Stroke Cerebrovasc Dis 2013; 22: 1018-1027

[100] Munoz-Venturelli P, Wang X, Lavados PM et al. Prophylactic heparin in acute intracerebral hemorrhage: a propensity score-matched analysis of the INTERACT2 study. Int J Stroke 2016; 11: 549-556

[101] Dennis M, Sandercock PA, Reid J et al. Effectiveness of thigh-length graduated compression stockings to reduce the risk of deep vein thrombosis after stroke (CLOTS trial 1): A multicentre, randomised controlled trial. Lancet 2009; 373: 1958-1965

[102] Dennis M, Sandercock P, Reid J et al. Effectiveness of intermittent pneumatic compression in reduction of risk of deep vein thrombosis in patients who have had a stroke (CLOTS 3): A multicentre randomised controlled trial. Lancet 2013; 382: 516-524 